\title{
Aplicação de Learning Analitycs ao Design Instrucional
}

\author{
Atylla Gomes dos Santos ${ }^{12}$, Isabel Dillmann Nunes ${ }^{2}$, Ulrich Schiel ${ }^{1}$ \\ ${ }^{1}$ Departamento de Sistemas e Computação - Universidade Federal de Campina Grande \\ Caixa Postal 10.106 - 58.429-900 - Campina Grande-PB - Brasil \\ ${ }^{2}$ Curso de Sistemas de Informação - FACISA/CESED \\ Av. Argemiro de Figueiredo 1901, Itararé - 58.411-020 - Campina Grande-PB - Brasil \\ \{atylla.gomes, beldillnunes\}@gmail.com, ulrich@computação.ufcg.edu.br

\begin{abstract}
The Instructional Design must be constantly evaluated and improved to address the needs of students. This article describes the application of Learning Analytics to Instructional Design in order to identify which activities or paths made by the students of the course that should be improved. A case study is conducted in a fictional course using simulated students, with 16 profiles specified with different characteristics. The simulation allowed us to identify which points of ID to be changed to cater for all profiles.
\end{abstract} \\ Resumo. O Design Instrucional deve ser constantemente avaliado e adaptado \\ para atender às necessidades dos estudantes. Este artigo descreve a aplicação \\ de Learning Analytics ao Design Instrucional - DI com o intuito de identificar \\ quais as atividades ou caminhos do curso realizados pelos alunos devem ser \\ melhorados. Um estudo de caso é realizado em um curso fictício utilizando \\ simulação de estudantes, sendo 16 perfis especificados com diferentes \\ características. A simulação permitiu identificar quais os pontos do DI devem \\ ser alterados para atender a todos os perfis.
}

\section{Introdução}

E-learning é uma união entre tecnologia e educação com o objetivo de permitir a construção do conhecimento com suporte de ferramentas baseadas na web. Esta definição também é conhecida por Educação On-line [Bittencourt e Costa 2010].

A construção de um ambiente de Educação On-line é composta pelas seguintes fases [Fetaji and Fetaji 2010]: Planejamento com a medida de indicadores iniciais, tais como habilidades computacionais, estilos de aprendizagem e níveis de motivação. Os resultados obtidos influenciam todas as outras fases; Design em que questões de personalização, técnicas pedagógicas e instrucionais são especificados; Implementação quando os cursos são realizados; Avaliação com o intuito de obter os resultados e Análise onde se devem aprimorar os resultados e propor iniciativas para melhoramentos dos cursos.

A fase de design instrucional, por ser determinante na aplicação do conhecimento e por considerar as abordagens pedagógicas existentes, é importante no processo do ambiente de aprendizagem. O Design Instrucional - DI está relacionado com o planejamento, desenvolvimento, implementação e a avaliação da aprendizagem [Dallacosta et al. 2010]. 
A Rede de Atividades de Alto Nível - RAAN é uma notação que traz as características necessárias para atender aos requisitos do planejamento de Design Instrucional e seu acompanhamento em tempo de execução. A RAAN utiliza como base formal a Rede de Projeto de Alto Nível - RPr, uma classe especial de Redes de Petri Colorida [Nunes e Schiel 2011].

A RAAN permite especificar atividades e o estado de indivíduos e grupos em relação a essas atividades. Tais características são fundamentais em um processo de Design Instrucional. As alterações para auxiliar alunos em risco, como também para corrigir falhas no DI podem ser realizadas em tempo de execução, determinando o que é chamado de rede adaptativa.

O acompanhamento das atividades em tempo de execução também permite armazenar detalhes sobre a execução do DI ao longo do tempo, criando um histórico. $\mathrm{O}$ objetivo deste artigo é mostrar que o histórico pode ser analisado por algoritmos de Learning Analytics [Elias 2011], produzindo informações pertinentes à melhoria de um Design Instrucional.

O artigo apresenta a Rede de Atividades de Alto Nível na seção 2. A seção 3 mostra a aplicação de Learning Analytics ao Design Instrucional e a seção 4 apresenta o Estudo de Caso realizado e seus resultados. Por fim, as conclusões são discutidas na seção 5 .

\section{Rede de Atividades de Alto Nível}

A Rede de Atividades - RA é um modelo gráfico inicialmente usado para planejar e acompanhar o processo de desenvolvimento de um projeto. Ela é uma rede com notação mais expressiva do que Redes de Petri convencionais, pois contêm elementos de representação de atividades compostas, eventos e repositório de artefatos, além de cada atividade possuir atributos de custo e tempo [Farias, 2008]. Assim como uma Rede de Petri ela representa um grafo dirigido bipartido e, além disso, esse grafo deve ser uma ordem parcial com um único nó de partida, chamado de begin e um nó de finalização, chamado de end.

Em analogia a Redes de Petri de Alto Nivel, as Redes de Atividades de Alto Nível permitem modelar a execução de uma rede por diversos elementos individualizados sujeitos a restrições de transição. RAs de Alto Nível podem ser aplicadas a qualquer processo dirigido a um objetivo a ser alcançado, ou seja, que visa chegar a um final específico, tal como um Design Instrucional. Permitem modelar um processo realizado por diversos indivíduos ou grupos simultaneamente. Nesse caso, diversas propriedades que antes eram associados a uma atividade como um todo agora são individualizadas para cada grupo ou indivíduo que a está executando.

Exemplo de Rede de Atividades de Alto Nível pode ser visualizado na Figura 1, a qual representa um curso dividido em 5 módulos. Cada módulo é uma sub-rede que não está aqui representada. Após a avaliação, o aluno possui duas possibilidades, ou a emissão do certificado caso tenho conseguido alcançar a nota 7,0 ou a realização de uma nova avaliação caso não tenha alcançado a nota exigida. 


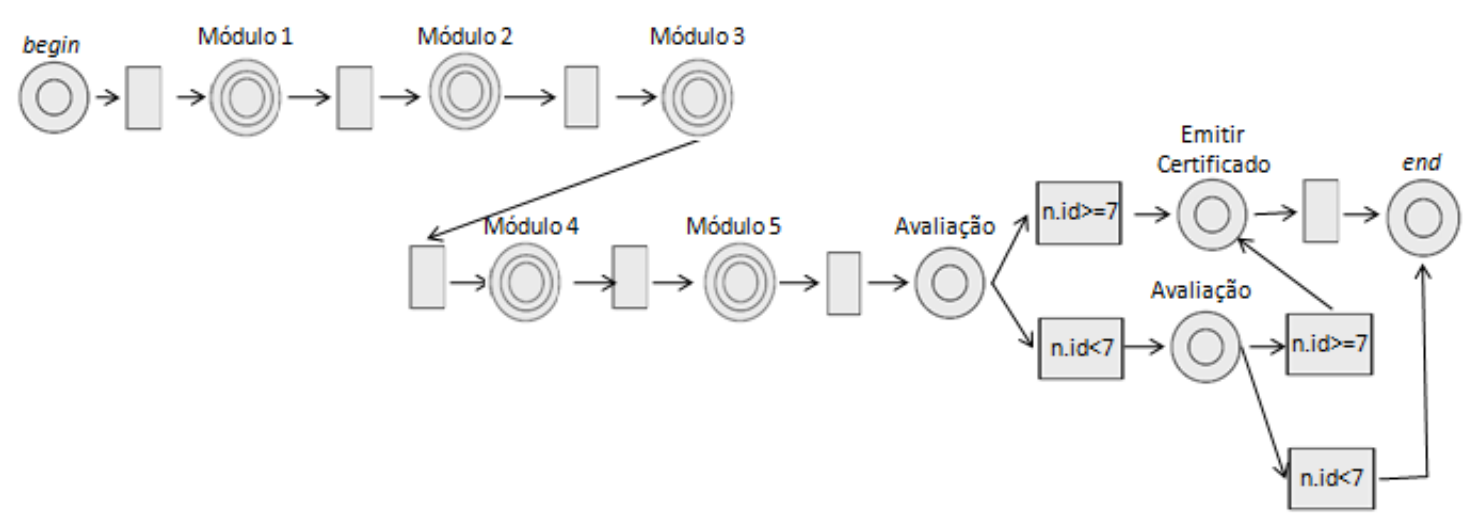

Figura 1. Rede de Atividades de Alto Nível do Curso de Secretariado

\section{Learning Analytics aplicado ao Design Instrucional}

O planejamento de uma disciplina é realizado no processo de Design Instrucional [Smith and Ragan 1999]. Na atividade de análise deve-se conhecer os alunos, seu contexto e as atividades que mais se adaptam ao seu perfil. A partir disso, a estratégia de entrega, da organização do curso e a forma como será gerenciado deve ser definido. Por fim, uma avaliação formativa ocorre e a revisão das instruções é realizada.

Técnicas de Learning Analytics permitem, além de analisar informações sobre o desempenho de alunos, extrair e analisar informações sobre o andamento de uma disciplina. A partir das análises realizadas nos dados do perfil do estudante, do modelo de Design Instrucional definido e nas informações de aprendizagem produzidas durante o aprendizado, o professor/tutor pode aplicar melhorias em sua disciplina.

A análise realizada pela técnica de Learning Analytics neste trabalho é baseada no SSA - Predictive Student Success Algorithm [Arnold and Pistilli 2012; Arnold et al. 2010], porém, aplicada sobre o histórico de Designs Instrucionais. O SSA é um modelo analítico preditivo que contém elementos do sistema acadêmico como também sobre o estudante, sendo considerado um modelo comportamental. Detalhes sobre o SSA não são possíveis de serem obtidos devido ao seu perfil de software comercial.

O algoritmo SSA prediz estudantes de risco a partir de quatro componentes: Performance, Esforço, Histórico e Características do Estudante.

Adaptando à proposta do trabalho de análise de informações sobre o Design Instrucional, pode-se identificar DIs de risco ou de sucesso. A Tabela 1 mostra a adaptação dos componentes de SSA aplicados ao DI.

\section{Estudo de Caso}

A partir da necessidade da realização de avaliação da proposta e a dificuldade de acesso às informações reais de aprendizagem, optou-se pela utilização de simulação de estudantes. A simulação apresentada é um método estocástico em que é possível definir o perfil de aprendizagem dos estudantes como também o perfil de ensino das atividades a serem entregues durante o curso. 
Tabela 1. Adaptação do modelo SSA para Design Instrucional

\begin{tabular}{|c|c|c|}
\hline Componente & Aplicado aos alunos & Aplicado do Design Instrucional \\
\hline Performance & $\begin{array}{c}\text { Medida a partir dos pontos } \\
\text { ganhos no curso. }\end{array}$ & $\begin{array}{c}\text { Medida por meio da realização de } \\
\text { atividades dentro/fora do tempo } \\
\text { previsto, baixa/alta adaptação do DI } \\
\text { e baixo/alto número de acessos às } \\
\text { atividades. }\end{array}$ \\
\hline Esforço & $\begin{array}{c}\text { Medido pela interação com os } \\
\text { Ambientes Virtuais de } \\
\text { Aprendizagem e comparado } \\
\text { com o esforço de seus colegas. }\end{array}$ & $\begin{array}{c}\text { Medido por meio da comparação } \\
\text { com outros DIs com objetivos } \\
\text { semelhantes. }\end{array}$ \\
\hline Histórico & $\begin{array}{c}\text { Medido por performance em } \\
\text { cursos preparatórios, notas no } \\
\text { ensino médio e testes. }\end{array}$ & $\begin{array}{c}\text { Medido pela performance do DI em } \\
\text { turmas anteriores. }\end{array}$ \\
\hline Características & $\begin{array}{c}\text { Tal como idade e local de } \\
\text { residência. }\end{array}$ & $\begin{array}{c}\text { Tal como data, público alvo e região } \\
\text { de aplicação. }\end{array}$ \\
\hline
\end{tabular}

\subsection{Simulação de Estudantes}

A ferramenta proposta por Dorça (2012) chamada de Simulador de Aprendizagem foi utilizada como base para a construção da ferramenta Simulador de Estudantes.

Um ponto a ressaltar é que o Simulador de Aprendizagem utiliza conceitos como um conjunto de uma ou mais atividades. O Simulador de Estudantes necessita que cada atividade seja especificada individualmente. Assim, neste trabalho, é utilizada diretamente a simulação da execução de cada atividade por parte dos alunos e não de um conceito como um todo.

O Simulador de Estudantes possui características que devem ser ressaltadas, tais como: o sequenciamento das atividades é definido pelo professor; o nível cognitivo de cada atividade também é definido (as atividades podem ser 0-Conhecimento, 1Compreensão, 2-Aplicação, 3-Análise, 4-Síntese e 5-Avaliação); o reforço para realização das atividades é calculado estocasticamente e o conceito de reprovado ou aprovado está diretamente ligado ao número de tentativas de execução de cada atividade (neste trabalho, o número de tentativas permitido - aprovado - é o dobro do nível cognitivo definido. Por exemplo, se o nível cognitivo é avaliação, seu valor é considerado 5 e portanto o aluno possui no máximo 12 tentativas).

A definição prévia dos estilos de aprendizagem das atividades exige que o professor tenha conhecimento do tipo de atividade a ser realizada. Assim, segundo o modelo de Felder e Silverman [Felder and Silverman 1988], tem-se os Estilos de Ensino de cada atividade.

As dimensões consideradas para o Estilo de Ensino são: Dimensão de Conteúdo - Qual o tipo de informação é enfatizada pelo professor? Concreta: fato. Abstrata: conceitual, teórico; Dimensão de Apresentação - Qual tipo de informação o professor utiliza mais? Visual: figuras, diagramas, filmes, demonstrações. Verbal: palestras, leituras, discussões; Dimensão de Participação do Estudante - Qual o modo de participação do estudante é facilitada pela apresentação? Ativo: estudante dialoga, reflete, se movimenta. Passivo: estudantes olham e escutam e Dimensão da Perspectiva 
- Que tipo de perspectiva é fornecida na informação apresentada? Sequencial: progressão passo a passo (tipo árvores). Global: contexto e relevância.

A Tabela 2 mostra os Estilos de Ensino e sua correspondência com os Estilos de Aprendizagem.

Tabela 2. Definição dos Estilos de Aprendizagem e Estilos de Ensino

\begin{tabular}{|c|c|c|c|c|}
\hline $\begin{array}{c}\text { Dimensão } \\
\text { (Aprendizagem) }\end{array}$ & Tipo & $\begin{array}{l}\text { Definição na } \\
\text { ferramenta }\end{array}$ & Tipo & $\begin{array}{c}\text { Dimensão } \\
\text { (Ensino) }\end{array}$ \\
\hline \multirow{2}{*}{ Processamento } & Ativo & 0 & Ativo & \multirow{2}{*}{$\begin{array}{l}\text { Participação } \\
\text { do Estudante }\end{array}$} \\
\hline & Reflexivo & 1 & Passivo & \\
\hline \multirow{2}{*}{ Percepção } & Sensitivo & 0 & Concreto & \multirow{2}{*}{ Conteúdo } \\
\hline & Intuitivo & 1 & Abstrato & \\
\hline \multirow{2}{*}{ Entrada } & Visual & 0 & Visual & \multirow{2}{*}{ Apresentação } \\
\hline & Verbal & 1 & Verbal & \\
\hline \multirow{2}{*}{ Organização } & Sequencial & 0 & Sequencial & \multirow{2}{*}{ Perspectiva } \\
\hline & Global & 1 & Global & \\
\hline
\end{tabular}

O Perfil do Estudante é definido a partir da combinação das 4 dimensões definidas no Estilo de Aprendizagem [Felder and Silverman 1988]. No total foram definidos 16 perfis (combinação de 2 possibilidades para cada um dos 4 perfis), que são: P1 (ativo, sensitivo, visual e sequencial), P2 (reflexivo, sensitivo, visual e sequencial), P3 (ativo, intuitivo, visual e sequencial), P4 (ativo, sensitivo, verbal e sequencial), P5 (ativo, sensitivo, visual e global), P6 (reflexivo, intuitivo, visual e sequencial), P7 (ativo, intuitivo, verbal e sequencial), P8 (ativo, sensitivo, verbal e global), P9 (reflexivo, sensitivo, verbal e sequencial), P10 (reflexivo, sensitivo, visual e global), P11 (ativo, intuitivo, visual e global), P12 (reflexivo, intuitivo, verbal e sequencial), P13 (ativo, intuitivo, verbal e global), P14 (reflexivo, intuitivo, visual e global), P15 (reflexivo, sensitivo, verbal e global) e P16 (reflexivo, intuitivo, verbal e global).

A avaliação do Estudo de Caso foi realizado por meio de 20 execuções de cada perfil em cada caminho possível do curso. Essa repetição foi necessária, pois é possível observar o comportamento do mesmo perfil de aluno em um mesmo curso, mas com possibilidade de reforços diferentes.

Devido ao processo de simulação, a avaliação realizada considerou somente componente Performance do algoritmo SSA aplicado ao Design Instrucional. Os demais componentes necessitam de informações históricas e/ou outros cursos.

\subsection{Avaliação do Curso}

Esta seção apresenta um curso fictício que contém atividades alternativas e paralelas. As atividades são definidas baseando-se em atividades realizadas por cursos reais à distância. A proposta desse curso tem o intuito de verificar como diferentes perfis de alunos se comportam por caminhos diferentes na execução de um mesmo curso. 
O curso possui 4 fases a serem realizadas pelos alunos e cada fase pode ter mais de um caminho a ser executado, nomeados de caminhos C1 a C8, como mostra o Design Instrucional da Figura 2.

A partir da avaliação realizada o caminho a ser considerado mais adequado é o caminho C1 (Figura 3), em que 12 dos 16 perfis conseguiram aprovação. Uma atenção especial deve ser dada aos perfis P1, P2, P5 e P10 que não se adequaram em nenhum caminho proporcionado pelo curso. Para tais perfis é necessária a proposta de um curso totalmente voltado para suas características, que possuem em comum serem sensitivos e visuais, o que não é atendido pelas atividades propostas nos 8 caminhos do curso.

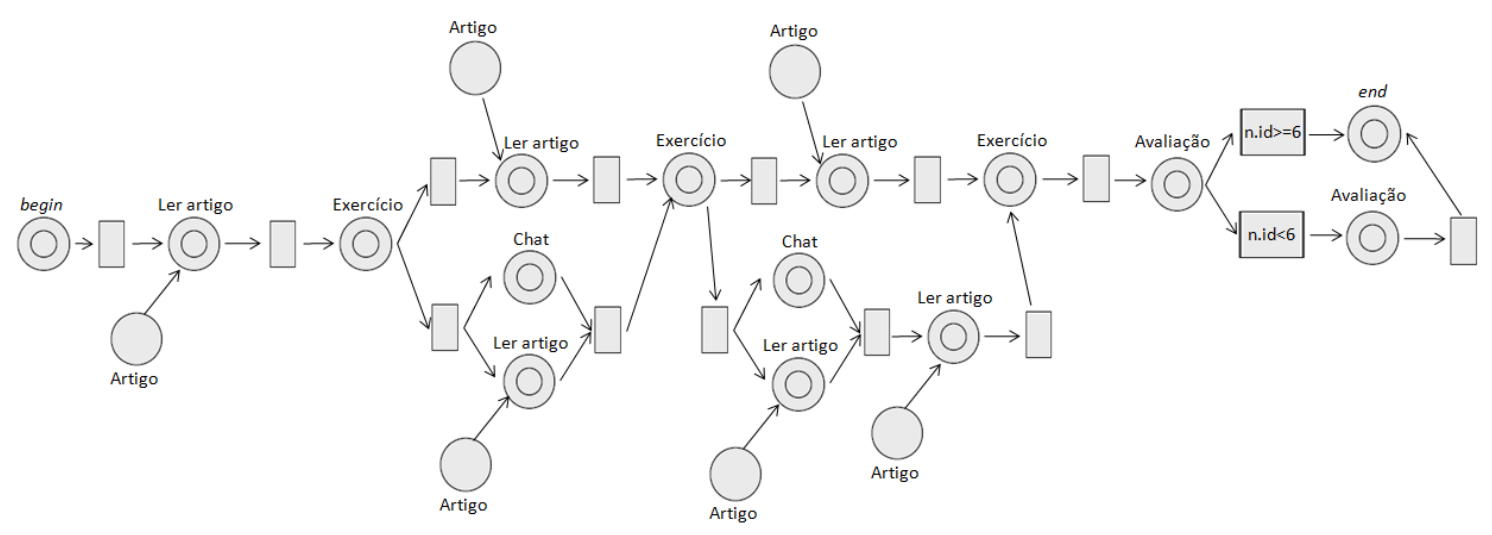

Figura 2. Rede de Atividades de Alto Nível do Curso Fictício.

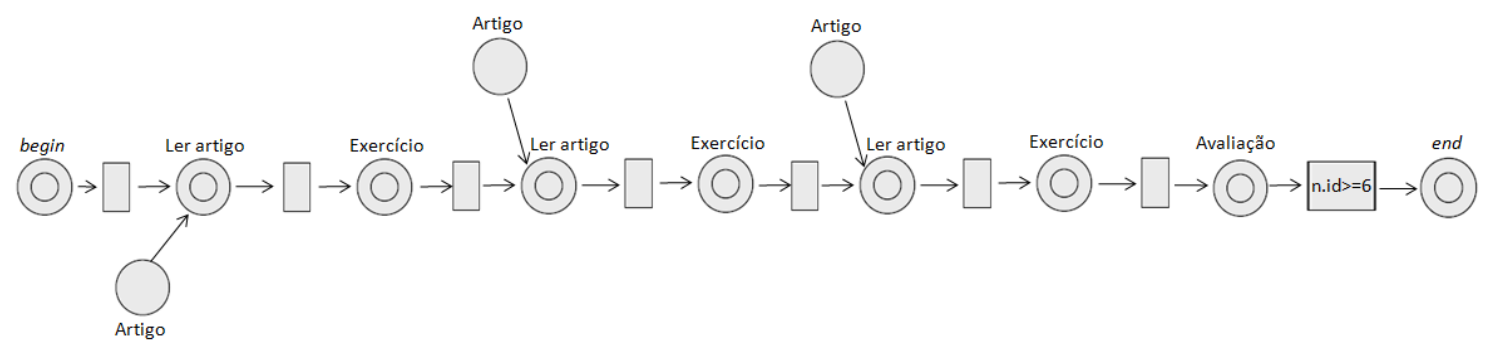

Figura 3. Caminho C1 do Curso Fictício

Com o intuito de atender aos perfis que não conseguiram aprovação no curso, e da análise realizada nos caminhos projetados, tem-se:

-Atividades c0 e c1 foram as que possuíram maior número de reprovações e devem ser alteradas;

-O caminho $\mathrm{C} 1$ foi o que obteve o maior número de aprovações, sendo considerado para a melhoria do curso.

O Caminho $\mathrm{C} 1$ é então alterado em suas atividades c0 e c1, sendo agora denominado de C1-2 (como mostra a Figura 4), da seguinte forma:

-Atividade c0 - De "Ler Artigo" é substituída por "Assistir Vídeo";

-Atividade c1 - De "Exercícios" é substituída por "Resolução de Problemas Práticos". 


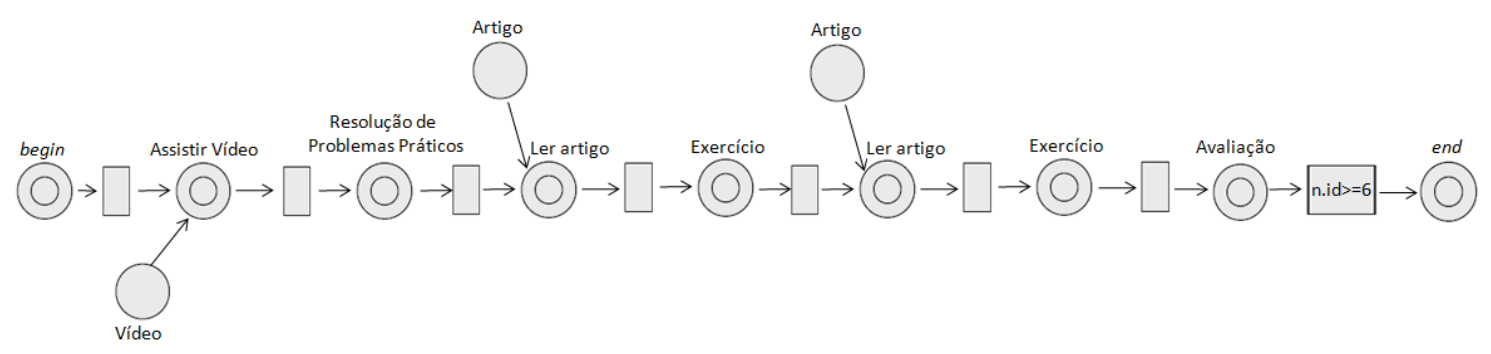

Figura 4. Rede de Atividades de Alto Nível do caminho C1-2

Os resultados obtidos foram:

-Perfil de aluno P5 foi aprovado;

-Perfil P10 conseguiu avançar no curso, porém ainda não foi aprovado;

-P1 e P2 foram reprovados.

Assim, o caminho C1-2 conseguiu atingir às necessidades e características do perfil de aluno P5, porém ainda deve ser alterado para atingir aos outros perfis.

Observando o número de iterações necessárias para realizar as atividades dos perfis $\mathrm{P} 1, \mathrm{P} 2$ e $\mathrm{P} 10$, tem-se que:

-Perfil de aluno P1 foi reprovado nas atividades c2 e c4;

-Perfil de aluno P2 foi reprovado na atividade c5;

-Perfil P10 foi reprovado nas atividades c3, c5 e c6.

As atividades que causaram a reprovação dos perfis em análise devem ser alteradas para atender às características de cada perfil. Sendo assim, o caminho C1-3 é proposto como mostra a Figura 5. Todas as atividades de exercícios e avaliação são propostas como "Resolução de Problemas Práticos" e todas as atividades de "Leitura de Artigo" são substituídas por "Assistir Vídeo".

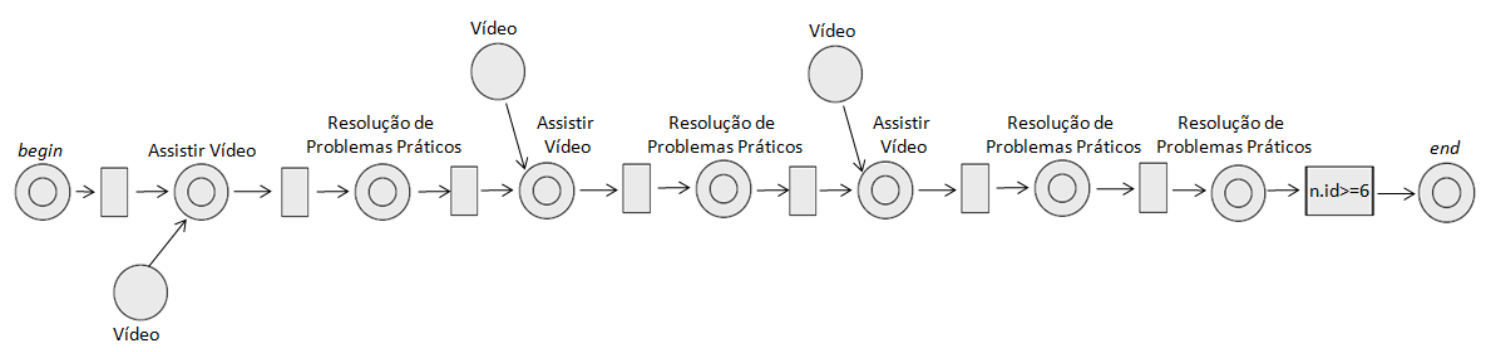

Figura 5. Rede de Atividades de Alto Nível do caminho C1-3.

Os resultados obtidos foram que todos os perfis em análise (P1, P2, P5 e P10) foram aprovados a partir das mudanças realizadas.

Os caminhos C1-2 e C1-3 conseguiram mostrar o avanço nas melhorias de um determinado caminho com o objetivo de atender às características de perfis que não conseguiram alcançar os resultados esperados. Porém, tais caminhos, devido à mudança do Estilo de Aprendizagem das atividades se tornam inadequados para outros perfis, que são: P8, P13 e P14. 
O caminho C1-3 atingiu um número maior de perfis aprovados em relação ao caminho $\mathrm{C} 1$ inicial, de 12 para 13 perfis aprovados. Porém, é necessário observar que cada caminho proposto possui características diferenciadas em relação ao Estilo de Aprendizagem de cada atividade. Isso faz com que os perfis de alunos se adéquam melhor a um determinado caminho, em detrimento a outro.

Os perfis de alunos, de P1 a P16, obtiveram aprovação em mais de um caminho. Considerando que o número de acessos é um dos fatores de Learning Analytics utilizado neste trabalho, é possível identificar o caminho com menor esforço para um determinado perfil de aluno.

A Tabela 3 indica o número de acessos por caminho em que um perfil de aluno foi aprovado. $\mathrm{O}$ número 1 indica que o aluno realizou o número mínimo de acessos para a realização de cada atividade do caminho, à medida que o número cresce, o número de acessos é maior. Os valores em destaque são os considerados como os melhores caminhos para cada perfil.

A partir dos valores da Tabela 3, pode-se identificar os melhores caminhos para cada perfil. A Tabela 4 relaciona o caminho mais adequado para cada perfil de aluno considerando o menor número de acessos para realização das atividades, sendo possível observar que o Curso Fictício conseguiu atingir aos 16 perfis de alunos do estudo.

Tabela 3. Número de acessos de cada perfil por caminho que obteve aprovação.

\begin{tabular}{|c|c|c|c|c|c|c|c|c|c|c|c|c|c|c|c|c|}
\hline & P1 & P2 & P3 & P4 & P5 & P6 & P7 & P8 & P9 & P10 & P11 & P12 & P13 & P14 & P15 & P16 \\
\hline $\mathrm{C} 1$ & & & 1,162 & 1,115 & & 1,353 & 1,033 & 1,227 & 1,202 & & 1,288 & 1 & 1 & 1,728 & 1,653 & 1,188333 \\
\hline C1-2 & & & & 1,133 & 1,725 & 1,39 & & & 1,192 & & 1,402 & 1,065 & & & 1,548 & 1,325 \\
\hline C1-3 & 1 & 11,358 & 1,325 & 1,08 & 1,227 & 1,692 & 1,175 & & 1 & 1,698 & 1,723 & 1,28 & & & 1,225 & 1,725 \\
\hline $\mathrm{C} 2$ & & & & 1,137 & & & 1,081 & 1,187 & 1,257 & & 1,287 & 1,103 & 1 & & 1,519 & 1,187143 \\
\hline C3 & & & & & & & 1,141 & 1,331 & 1,254 & & 1,387 & 1,176 & 1,077 & & & 1,27 \\
\hline C4 & & & & 1,108 & & & 1,093 & 1,225 & 1,198 & & 1,335 & 1,165 & 1,014 & & 1,46 & 1,192857 \\
\hline C5 & & & & 1,133 & & 1,351 & 1,056 & 1,222 & 1,208 & & 1,272 & 1 & 1 & & 1,667 & 1,234722 \\
\hline C6 & & & & & & & 1,061 & 1,196 & 1,239 & & 1,299 & 1,089 & 1 & 1,676 & 1,551 & 1,189024 \\
\hline C7 & & & & & & & 1,056 & 1,234 & 1,213 & & 1,278 & 1,094 & 1,014 & & 1,558 & 1,177907 \\
\hline C8 & & & & 1,092 & & & 1,088 & 1,203 & 1,203 & & & 1,14 & 1,009 & 1,706 & 1,474 & 1,183333 \\
\hline
\end{tabular}

Tabela 4. Caminhos mais adequados para cada Perfil de aluno.

\begin{tabular}{|c|l|}
\hline Caminho & Perfis adequados \\
\hline C1 & P3 - P7 - P12-P13 \\
\hline C1-2 & - \\
\hline C1-3 & P1 - P2 - P4 - P5 - P9 - P10-P15 \\
\hline C2 & P8 \\
\hline C3 & - \\
\hline C4 & - \\
\hline C5 & P6 - P11-P12-P13 \\
\hline C6 & P13-P14 \\
\hline C7 & P16 \\
\hline C8 & - \\
\hline
\end{tabular}


Também é possível observar, a partir da Tabela 4, que existem caminhos que podem ser desconsiderados no Design Instrucional do Curso Fictício, pois não são se encaixam em nenhum dos melhores caminhos para os perfis de alunos. Outro ponto a considerar, é que existem perfis que tiveram seus melhores desempenhos em mais de um caminho, como por exemplo, o perfil P13, que teve seus melhores resultados nos caminhos $\mathrm{C} 1, \mathrm{C} 5$ e $\mathrm{C} 6$.

A Figura 6 mostra a nova proposta de Design Instrucional para o Curso Fictício considerando as análises realizadas com Learning Analytics e os resultados obtidos.

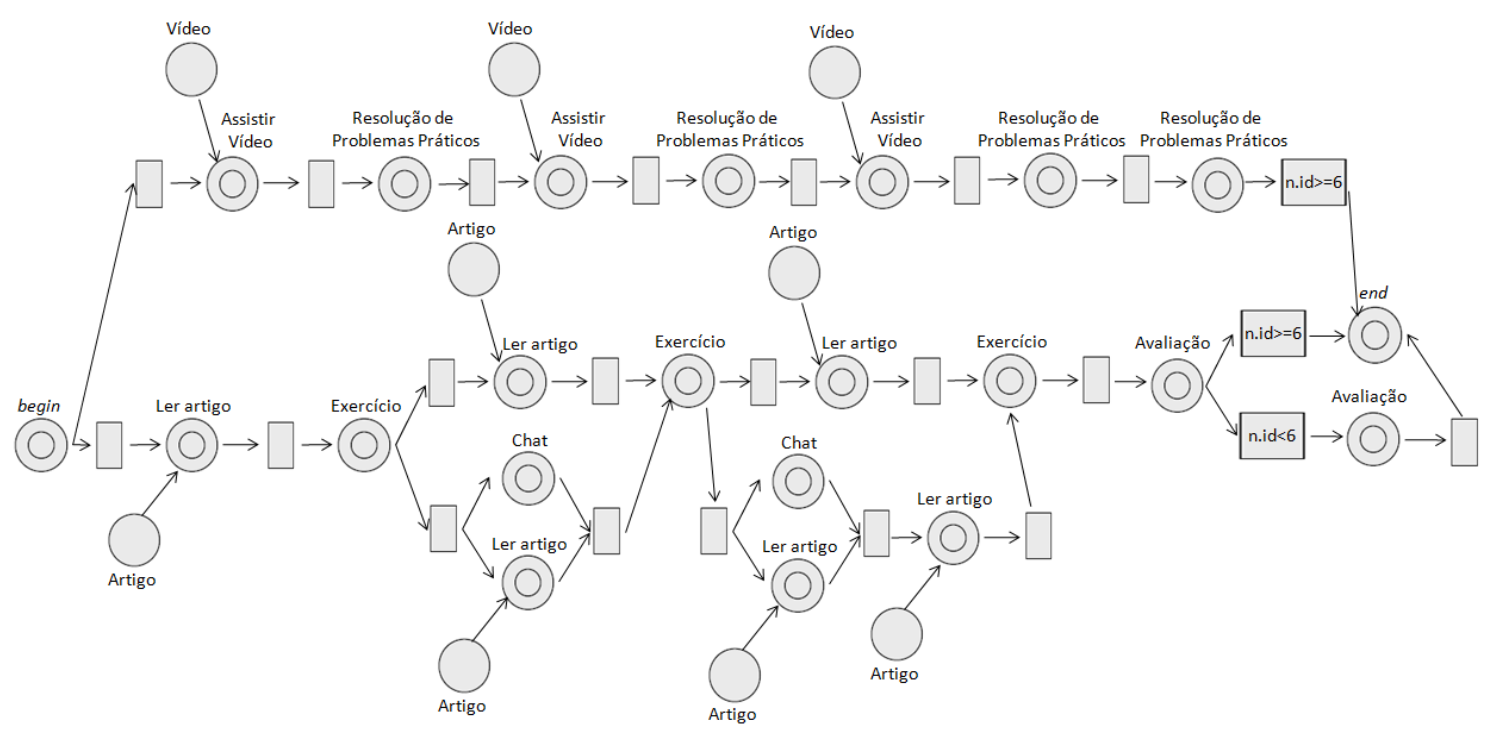

Figura 6. Rede de Atividades de Alto Nível do Curso Fictício depois da aplicação de Learning Analytics.

O DI apresentado na Figura 6, utilizando a notação de Rede de Atividades de Alto Nível, mostra a inserção do caminho C1-3 (para aprovação dos perfis de aluno P1, P2, P4, P5, P9, P10 e P15). Os caminhos C1-2, C3, C4 e C8 poderiam ser descartados no DI do curso, pois não são considerados como melhores caminhos para os perfis de alunos, porém eles não podem ser excluídos porque possuem trechos pertencentes a outros caminhos.

\section{Conclusões}

Os resultados obtidos com a aplicação dos algoritmos de Learning Analytics aos cursos utilizados nos Estudos de Casos, reais ou fictícios, introduzem informações relevantes para o melhoramento do Design Instrucional desses cursos como também de cursos em geral. A análise foi realizada em todo o curso, mas o mesmo estudo pode ser aplicado somente em módulos ou períodos que o professor/tutor considere com maior tendência a riscos.

A Simulação de Estudantes foi realizada baseando-se na Simulação de Aprendizagem proposta por Dorça [Dorça 2012] em sua tese de doutorado. A Simulação de Estudantes considera seu estilo de aprendizagem, o estilo de ensino das atividades, o nível cognitivo da atividade a ser alcançado pelo aluno e ainda o reforço necessário para o aluno alcançar o nível cognitivo. 
A Simulação de Estudantes permitiu a especificação de 16 diferentes perfis definidos a partir da combinação de: seus estilos de processamento da informação, tipo de informação que o aluno prefere receber (percepção), qual o canal sensorial que o aluno percebe as informações (entrada) e organização de realização do curso.

Trabalhos futuros ainda devem ser realizados, tal como o estudo e aplicação de formas de visualização dos dados obtidos e a consideração de características do perfil dos alunos que não foram consideradas, tais como idade, sexo e escolaridade.

\section{Referências Bibliográficas}

Arnold, K.E., Tanes, Z. \& King, A.S. (2010) “Administrative perceptions of datamining software Signals: Promoting student success and retention". The Journal of Academic Administration in Higher Education 6 (2), 29-39.

Arnold, K. E. and Pistilli, M. D. (2012) "Course Signals at Purdue: Using learning analytics to increase student success". In: Proceedings of the 2nd International Conference on Learning Analytics \& Knowledge. New York: ACM.

Bittencourt, I. I. e Costa, E. (2010) "Modelos e Ferramentas para Construção de Sistemas Educacionais Adaptativos e Semânticos". In: XXI Simpósio Brasileiro de Informática na Educação. João Pessoa - PB.

Dallacosta, A.; Cazetta, G.; Souza, S. G de. (2010) "Novas tecnologias aplicadas na elaboração de material instrucional online". In: $3^{\circ}$ Simpósio Hipertexto e Tecnologias na Educação (redes sociais e aprendizagem). Recife - PE.

Dorça, F. A. (2012) "Uma abordagem estocástica baseada em Aprendizagem por Reforço para modelagem automática e dinâmica de Estilos de Aprendizagem de Estudantes em Sistemas Adaptativos e Inteligentes para Educação a Distância”. Tese de Doutorado. Faculdade de Engenharia Elétrica da Universidade Federal de Uberlândia.

Elias, T. (2011) “Learning Analytics: Definitions, Processes and Potential”. Disponível em: http://learninganalytics.net/LearningAnalyticsDefinitionsProcesses Potential.pdf.

Farias, C. B de A. (2008) "Uma extensão de Rede de Petri para Modelagem de Processos e Controle de Projetos". Tese de Doutorado, Coordenação de PósGraduação em Engenharia Elétrica - UFCG. Campina Grande. 281 pág.

Felder, R. M. and Silverman, L. K. (1988) "Learning and Teaching Styles". In Engineering Education. Engr. Education, 78(7), 674-681.

Fetaji, B; Fetaji, M. (2010) "E-Learning Indicators: A Multidimensional Model for Planning Developing and Evaluating E-Learning Software Solutions". In: ELearning, experiences and future. Edited by Safeeullah Soomro and published by InTeh. Pag 1- 34.

Nunes, I. D. ; Schiel, U. (2011) "Design Instrucional e seu acompanhamento em tempo real utilizando Rede de Atividades". In: Simpósio Brasileiro de Informática na Educação, 2011, Aracajú. Simpósio Brasileiro de Informática na Educação.

Smith, P. L. and Ragan, T. J. (1999) “Instructional Design”. Second Edition. John Wiley \& Sons, Incorporated. 